\title{
Validation of the Chronic Tinnitus Acceptance Questionnaire (CTAQ-I): the Italian version
}

\section{Validazione della versione italiana del Questionario sull'Accettazione dell'Acufene Cronico (CTAQ-I)}

R. MOSCHEN ${ }^{1}$, A. FIORETTI ${ }^{2}$, A. EIBENSTEIN² ${ }^{2}$, E. NATALINI², G. CHIARELLA ${ }^{3}$, P. VIOLA ${ }^{3}$, D. CUDA ${ }^{4}$, C. CASSANDRO ${ }^{5}$, A. SCARPA ${ }^{6}$, G. RUMPOLD ${ }^{1}$, D. RIEDL ${ }^{1}$

${ }^{1}$ University Clinic of Medical Psychology, Medical University of Innsbruck, Austria; ${ }^{2}$ Tinnitus Center, European Hospital, Rome, Italy: ${ }^{3}$ Department of Experimental and Clinical Medicine, Unit of Audiology and Phoniatrics, University "Magna Graecia" of Catanzaro, Italy; ${ }^{4}$ Department of Otorhinolaryngology, "Guglielmo da Saliceto" Hospital, Piacenza, Italy; ${ }^{5}$ ENT Department, "San Giovanni Battista" Hospital, University of Torino, Italy; ${ }^{6}$ UO Otorinolaringoiatria - AOU "San Giovanni di Dio e Ruggi d'Aragona" of Salerno, Italy

\section{SUMMARY}

Tinnitus is a widespread symptom that is chronically experienced by approximately $10 \%$ of the adult population. While the vast majority of individuals do not feel impaired by their ear noise, about $0.5-3 \%$ of the adult population develops severe distress and feels impaired in everyday life. The severity of the distress experienced can only partially be explained by the characteristics of the ear noise itself (e.g. objective tinnitus loudness or duration). Psychological variables such as tinnitus acceptance are being increasingly investigated by tinnitus research. The aim of this study was to analyse the psychometric quality of the Italian version of the "Chronic Tinnitus Acceptance Questionnaire" (CTAQ-I). The CTAQ-I is an adaption of the Italian "Chronic Pain Acceptance Questionnaire (CPAQ)" and altered for the study of tinnitus acceptance. This cross-sectional multicentre questionnaire study included 141 outpatients with chronic tinnitus. Sociodemographic and clinical data were assessed with the Tinnitus Sample Case History (TSCH), tinnitus acceptance with the Italian "Chronic Tinnitus Acceptance Questionnaire" (CTAQ-I), tinnitus distress with the Tinnitus Handicap Inventory (THI) and the Tinnitus Questionnaire Short Form (TQ 12-I). Furthermore, we assessed the patient's general psychological distress (Brief Symptom Inventory - BSI) and Health Related Quality of Life (SF-36). Psychometric and factorial evaluation of the CTAQ-I were carried out. To investigate the influence of sociodemographic and numerous clinical variables on tinnitus acceptance we calculated correlation coefficients, analysis of variance and independent sample-t-tests. The factor analysis revealed a two-factor solution ("activity engagement" and "tinnitus willingness"), accounting for $41.1 \%$ of the variance. Good internal consistency for the total score $(\alpha=0.84)$ and both factors $(\alpha=0.80-0.88)$ was found. Moderate negative correlations with tinnitus distress indicated good validity. Tinnitus acceptance was further correlated with lower psychological distress and subjective tinnitus loudness, as well as increased emotional well-being. Tinnitus acceptance is considered to be an important source of therapeutic change in behavioural-based treatments as well as in professional counselling for tinnitus. Our study shows that the CTAQ-I is a reliable and valid instrument to measure the acceptance of patients suffering from chronic tinnitus.

KEY WORDS: Chronic Tinnitus Acceptance Questionnaire • Acceptance • Tinnitus • Questionnaire • Italian • Validation

\section{RIASSUNTO}

L'acufene è un sintomo ampiamente diffuso, rappresenta una condizione cronica per circa il 10\% della popolazione adulta. La maggioranza dei pazienti non si sente danneggiata dall'acufene, ma circa lo 0,5-3\% della popolazione adulta sviluppa un grave disagio e si sente compromesso nella vita di tutti i giorni. Quanto grave sia il disagio provato può essere solo parzialmente spiegato dalle caratteristiche dell'acufene stesso (come intensità o durata). Le variabili psicologiche, come l'accettazione dell'acufene, sono sempre più indagate dalla ricerca. Lo scopo di questo studio è analizzare la qualità psicometrica della versione italiana del Chronic Tinnitus Acceptance Questionnaire, "Questionario sull'accettazione dell'acufene cronico" (CTAQ-I). Il CTAQ-I è un adattamento del questionario italiano sull' accettazione del dolore cronico (CPAQ), adattato per lo scopo dello studio dell'acufene cronico. Studio multicentrico trasversale mediante questionario su 141 pazienti ambulatoriali con acufene cronico. I dati sociodemografici e clinici sono stati valutati con la Tinnitus Sample Case History (TSCH), l'accettazione dell'acufene con il "Questionario sull'accettazione dell'acufene cronico" (CTAQ-I), il disagio dell'acufene con la Tinnitus Handicap Inventory (THI) e il mini Questionnaire (TQ 12-I). Inoltre abbiamo valutato il disagio psicologico generale del paziente (Brief Symptom Inventory - BSI) e la qualità della vita correlata alla salute (SF-36). Sono state condotte valutazioni psicometriche e fattoriali del CTAQ-I. Per studiare l'influenza delle variabili sociodemografiche e numerose variabili cliniche sull'accettazione dell'acufene, abbiamo calcolato i coefficienti di correlazione, l'analisi della varianza e il test $T$ per campioni indipendenti. L'analisi fattoriale ha rivelato una soluzione a due fattori ('activity engagement' $e$ 'tinnitus willingness'), che rappresentano il 41,1\% della varianza. È stata trovata una buona coerenza interna per il punteggio totale $(\alpha=0,84)$ ed entrambi i fattori $(\alpha=0,80-0,88)$. Le moderate correlazioni negative con il disagio indicavano una buona validità. L'accettazione dell'acufene era ulteriormente correlata a un minore stress psicologico e minore volume dell'acufene soggettivo, oltre che ad un maggiore benessere emotivo. L'accettazione dell'acufene è considerata un'importante risorsa terapeutica di cambiamento nei trattamenti (comportamentali e di counseling professionale) per l'acufene. Il nostro studio mostra che il CTAQ-I è uno strumento affidabile e valido per misurare l'accettazione dell'acufene cronico da parte dei pazienti. 


\section{Introduction}

Tinnitus is defined as the perception of sound in the absence of a corresponding auditory source. Epidemiological studies report a prevalence of $10-16 \%$ for chronic tinnitus in the adult population, which increases with age ${ }^{12}$. While the vast majority of individuals are unaffected by tinnitus, $0.5-3 \%$ of the adult population report impairing tinnitus-associated symptoms of disturbed sleep and decreased cognitive functions, irritability, disruption of everyday activities as well as psychological problems such as anxiety and depressive symptoms ${ }^{34}$. Distressing tinnitus is often associated with audiological dysfunctions, noise sensitivity and impaired quality of life of patients and in many cases their relatives as well ${ }^{5-7}$.

The subjectively experienced strain due to tinnitus can only partially be explained by psychoacoustic parameters such as objective tinnitus loudness or duration ${ }^{8}$. A large body of literature suggests that tinnitus related cognitions (e.g. catastrophic thinking), emotions (e.g. anxiety and depression) and personality traits as well as coping strategies play an important role in tinnitus perception and distress ${ }^{9-12}$.

Since there is no specific pharmacological treatment available to cure chronic tinnitus ${ }^{13}$, at present therapeutic strategies support the process of coping with tinnitus, building stress management and treating comorbid symptoms. Cognitive behavioural interventions are, in addition to multimodal therapies, the most widely used treatment strategies ${ }^{13}$. Independent meta-analyses and systematic reviews revealed convincing evidence for the efficacy of cognitive behavioural therapies to reduce tinnitus-related distress ${ }^{14} 15$.

In the recent development of cognitive-behavioral treatments, acceptance- and mindfulness-based approaches, such as the "Acceptance and Commitment Therapy" or the "Mindfulness-Based Cognitive Therapy", have been integrated in the treatment of tinnitus ${ }^{16-18}$. These approaches are considered particularly relevant when dealing with a chronic problem, such as chronic tinnitus. There are a growing number of experiential, correlational and outcome studies showing that tinnitus related acceptance is related to better functioning and well-being ${ }^{18-22}$.

The word "acceptance" takes its roots from the Latin word "accipere" which means "admit, let in, hear, learn". Psychological acceptance is also described as a mindful and non-evaluative approach to internal experiences such as emotions, physical sensations or thoughts. Acceptance has been further described as the openness to experience thoughts and emotions as they are, while refraining from attempting to direct and actively change these situations, feelings, and thoughts. Psychological acceptance does not equate to an attitude of resignation or "giving up", but is characterised by an active shaping of the situation in the presence of tinnitus. Currently, it is not entirely clear which specific individual coping strategies are useful or maladaptive for long-term adaptation to chronic tinnitus. A useful model for the understanding of the tinnitus adaptation process and the value of acceptance is provided by the "dual-process model of coping" by Brandtstädter and Renner ${ }^{23}$. It distinguishes between two complementary coping strategies: accommodative and assimilative coping. The assimilative coping mode is characterised by active attempts to change or control a stressful situation, like a chronic burden or health impairment. This persistent way of pursuing one's goal can include activities such as acquiring relevant knowledge and skills, or using compensatory means (e.g. using external support sources such as hearing aids and sound generators to solve the problem). When a problem remains unresolved and blocks life goals, the level of stress usually increases and a transition from assimilative to accommodative coping may be required. Accommodative coping is the flexible adjustment of individual goals and preferences to situational constraints or limitations. This can involve the devaluation or reevaluation of a particular goal (e.g. the elimination of tinnitus), as well as the consideration of alternative feasible goals. The acceptance of adverse consequences and uncontrollability of an insoluble problem is often considered as a key process in accommodative coping.

For many patients, the word acceptance has a bad overtone because it means to give up the expectation of getting rid of their tinnitus. Patients often maintain a high level of activity to avoid "giving up" or passive suffering from tinnitus or are constantly struggling against it. This may also include a constant search for new treatments and at considerable personal and financial costs. These constant strong assimilative efforts toward a blocked target (i.e. the extinction of tinnitus) can lead to the exhaustion of one's own action resources and increased psychological distress.

On a theoretical level, acceptance is closely related to its opposite psychological process - experiential avoidance (EA). EA is defined as the avoidance of internal sensations, thoughts etc. Avoidance behaviour may intensify negative thoughts ${ }^{24}$ and is linked to more distressing tinnitus as well as increased anxiety and tinnitus-related distress. Patients suffering from chronic tinnitus often avoid specific situations, including silence as well as certain sounds that are perceived as annoying ${ }^{25}$. Some patients remove themselves from social relations to offer protect from noise pollution ${ }^{26}$ or to avoid high (sometimes po- 
tentially harmful) noise levels ${ }^{27}$. Another avoidance tactic used is to mask the tinnitus by persistently distracting oneself using background sounds like radio or TV-sets. Although EA is associated with short-term relief, in the long run it strongly impairs the patients' quality of life. While trying to protect oneself from a disabling tinnitus by avoiding its perception and associated emotions and thoughts, this may paradoxically lead to increased anxiety and tinnitus-related distress ${ }^{28}$.

Acceptance refers to a process by which the tinnitus and associated thoughts and feelings lose their functional influence on overt behaviour, since the target of control is changed from uncontrollable factors (i.e. the tinnitus itself) to more controllable factors (i.e. changing behaviour to increase daily function despite tinnitus).

In order to evaluate the efficacy of acceptance-orientated treatment, specific and validated instruments to measure acceptance are necessary. In the treatment of chronic pain, acceptance-oriented instruments such as the "Chronic Pain Acceptance Questionnaire" (CPAQ) have already been developed and validated. Due to various similarities often described between chronic pain and chronic tinnitus - both groups suffer from intractable sensory symptoms and demonstrate problems with aspects of focus/ attention, perceived lack of control over symptoms, catastrophic thinking and maladaptive coping strategie - the CPAQ has been adapted for tinnitus in English (Moreland 2009, unpublished thesis) as well as German ${ }^{29}$. To our knowledge no tinnitus acceptance questionnaire has been developed in Italian thus far.

Therefore, the aim of the present study was to evaluate the psychometric properties of the Italian CTAQ-I and to investigate its relationship to tinnitus distress, well-being and psychological distress.

\section{Materials and methods}

\section{Sample and setting}

The sample of this cross-sectional multicentre study consisted of 150 outpatients which were included from the following healthcare institutions: Tinnitus Centre, European Hospital (Rome), the Department of Otorhinolaryngology, "Guglielmo da Saliceto" Hospital (Piacenza), and the Department of Experimental and Clinical Medicine, Unit of Audiology and Phoniatrics, University "Magna Graecia" (Catanzaro). Patients were included in the study if they: (a) had tinnitus for at least 3 months, (b) were older than 18 years, (c) spoke Italian fluently and (d) had no apparent cognitive impairment. The patients completed the questionnaires as part of routine clinical practice.
All procedures performed in studies involving human participants were in accordance with the ethical standards of the institutional and/or national research committee and with the 1964 Helsinki declaration and its later amendments. The study was performed in three ENT centres in Italy, namely Rome, Catanzaro and Piacenza. Heads of these centres (MD Alberto Eibenstein, MD Giuseppe Chiarella and MD Domenico Cuda) agreed on the procedures and provided access to tinnitus patients. Participation was voluntary and each patient gave written consent before filling out the questionnaires.

\section{Measures}

\section{Tinnitus Sample Case History (TSCH)}

Sociodemographic and clinical data were assessed with the Italian version of the TSCH. It was developed by the Tinnitus Research Initiative as an attempt to standardise the assessment of sociodemographic and clinical data in tinnitus research ${ }^{30}$. The questionnaire consists of 35 items on background (i.e. age, gender), tinnitus history (i.e. loudness, pitch, percentage of awake time aware of tinnitus, hyperacusis) and related conditions (i.e. hearing impairment, noise annoyance, vertigo/dizziness).

\section{Chronic Tinnitus Acceptance Questionnaire - Italian version (CTAQ-I)}

The acceptance of chronic tinnitus was measured by the Italian version of the "Chronic Tinnitus Acceptance Questionnaire" (CTAQ-I). This is an adaption of the validated Italian version ${ }^{31}$ of the "Chronic Pain Acceptance Questionnaire" (CPAQ) ${ }^{32}$ for tinnitus, replacing the word "pain" with "tinnitus" or "ear noise". The CTAQ-I consists of 20 items, rated on a 7-point Likert scale $(0=$ never true, $6=$ always true $)$ for rating tinnitus acceptance. Eleven items measure the extent of participation in normal daily activities regardless of acknowledged tinnitus ("activity engagement", AE) and the remaining nine reverse-keyed items measure the willingness to have tinnitus present without trying to avoid or reduce it ("Tinnitus Willingness", TW). The CTAQ-I total score ranges from $0-120$, with a higher score indicating higher acceptance of tinnitus. A validation of the German version of the CTAQ (CTAQ-G) has shown an internal consistency of $\alpha=0.85^{29}$.

\section{Tinnitus Handicap Inventory (THI)}

The Tinnitus Handicap Inventory (THI) is a self-administered tool, widely used in tinnitus research and in clinical contexts, to assess the impact of the tinnitus in daily life. It consists of 25 items that can be divided in a functional, emotional and catastrophic subscale. Based on the total score tinnitus severity can be graded from slight (grade 1) 
to catastrophic (grade 5). Good reliability $(\alpha=0.94)$ and validity was reported for the total score of the Italian THI version ${ }^{33}$.

\section{Tinnitus Questionnaire (TQ 12)}

Tinnitus distress was also measured with the Italian 12 item short form of the Tinnitus Questionnaire (TQ 12-I) ${ }^{34}$. For the TQ 12-I, good reliability and validity were reported. The total score allows the classification of the patients as compensated (0-7), moderately distressed ${ }^{8-12}$, severely distressed ${ }^{13-18}$, and most severely distressed ${ }^{19-24}$.

\section{Brief Symptom Inventory (BSI)}

Mental health symptoms and psychological distress were assessed with the Brief Symptom Inventory. The BSI consists of 53 items to capture global psychological distress. Good reliability and validity for the subscales and total score were reported ${ }^{35}$.

\section{Short Form (36) Health Survey}

The health status was measured with the Short Form Health Survey (SF-36), a broadly used, well established instrument to assess the Health-Related Quality of Life (HRQOL). The SF-36 consists of 36 items which can be divided into a physical and psychological total score. The subscale's internal consistencies were tested in nine samples, showing a Cronbach's $\alpha$ between 0.77-0.93. Good validity has been reported in several studies ${ }^{36}$.

\section{Statistical analysis}

Psychometric values (means, standard deviations, itemtotal correlations) of the items are presented. A factor analyses (maximum likelihood, direct oblimin) with a fixed number of factors was conducted to investigate the proposed two-factor solution ${ }^{29}$. Scree plots and Eigenvalues were used to determine the ideal number of factors. Reliability was evaluated by calculating Cronbach's $\alpha$ (internal consistency) and item-total correlations. Validity was evaluated by two-sided Pearson correlations with the THI, TQ 12-I, BSI and SF-36 scores. Statistical analyses were conducted using IBM SPSS (ver. 22).

\section{Results}

Of the initial 150 patients, 4 (2.6\%) were excluded because their tinnitus lasted less than three months and another 5 patients $(3.3 \%)$ because of fully missing CTAQ data. The remaining 141 patients were included in the study of which 47.5\% were recruited in Piacenza, 37.6\% in Rome and 14.9\% in Catanzaro. Their mean age was 53.8 years and $55 \%$ were men. The mean tinnitus duration was 7.5 years and a large proportion of the sample reported tinnitus in both ears. More than half of the sample had subjective hear- ing problems and about a third reported physical discomfort because of surrounding sounds (hyperacusis). About a quarter of the sample had undergone several treatments due to their tinnitus. Overall, patients were aware of their tinnitus $(75.4 \%)$ and were annoyed $(48.9 \%)$ about their time spent awake over the last month. Based on the THI total score, tinnitus distress was graded as follows: $19.1 \%$ very mild, $25.5 \%$ mild, $31.9 \%$ moderate, $14.9 \%$ severe and $7.1 \%$ catastrophic. For details see Table I.

\section{Psychometric values}

The mean, standard deviation and item-total correlation of each item included are presented in Table II. The mean total score was 73.6 (SD: 20.1), the mean "activity engagement" score was 42.4 (SD: 14.4) and the mean "tinnitus willingness" score was 31.2 (SD: 11.8).

We tested the proposed factor solution ${ }^{29}$ by factor analysis (maximum likelihood). Bartlett's test of sphericity $\left(\chi^{2}\right.$ $(171)=1096.34, p<0.001)$ was significant and the Kaiser-Meyer-Olkin measure verified the sampling adequacy for the analysis $(\mathrm{KMO}=0.82)$. Initial Eigenvalues indicated that the two factors explained $41.1 \%$ of the variance (factor 1: $27.7 \%$, factor $2: 13.5 \%$ ). The diagonals of the anti-image correlation matrix were between 0.78 and 0.92 . We were able to reproduce the proposed two-factor solution using oblique rotation (oblimin). While the scree plot supported a two-factor-solution (Fig. 1), the Eigenvalues indicated a four-factor solution. An exploratory factor analysis (maximum likelihood) without a predefined number of factors resulted in a four-factor solution, explaining $50.5 \%$ of the variance. Yet, this solution contained multiple cross-loadings and an inconclusive factorial structure. In the further calculations, the two-factor solution was used. Table III shows the factor loadings for the two-factor solution after rotation.

Good internal consistency was found for the total score $(\alpha=0.84)$ as well as for the factor "activity engagement" $(\alpha=0.88)$ and the factor "tinnitus willingness" $(\alpha=0.80)$. The exclusion of item 3 would slightly improve the Cronbach's $\alpha$ for factor $1(\alpha=0.89)$, but not for the total score, while the exclusion of item 7 would improve the internal consistency of both factor $2(\alpha=0.81)$ and the total score $(\alpha=0.86)$. The item-total correlations were acceptable for all items, except item 3, 7, 11 and 16.

\section{Validity analysis}

For the CTAQ-I total score high negative correlations with both the TQ 12-I and THI total scores and the respective subscales were found, which indicated good convergent validity. Furthermore, elevated tinnitus acceptance was correlated negatively with higher global psychological 
Table I. Clinical properties of the sample.

\begin{tabular}{lc}
\hline TN loudness: 0-100 (SD) & $54.2(28.4)$ \\
Missing & $10(7.1 \%)$ \\
Duration: years (SD) & $7.5(10.4)$ \\
Family history of tinnitus complaints & $34(24.1 \%)$ \\
Missing & $1(0.7 \%)$ \\
Subjective hearing problem & $82(58.2 \%)$ \\
Missing & $1(0.7 \%)$ \\
Hearing aid & $19(13.6 \%)$ \\
Missing & $1(0.7 \%)$ \\
Hyperacusis & $53(37.6 \%)$ \\
Missing & $1(0.7 \%)$ \\
Tinnitus loudness varies during the day & \\
Yes & $94(66.7 \%)$ \\
No & $45(31.9 \%)$ \\
Missing & $2(1.4 \%)$ \\
Pulsatile tinnitus & $36(25.7 \%)$ \\
If yes: with heartbeat & $15(10.7 \%)$ \\
If yes: different from heartbeat & $21(14.9 \%)$ \\
Location & $20(14.2 \%)$ \\
Right ear & $6(4.3 \%)$ \\
Left ear & $2(1.4 \%)$ \\
Both ears & \\
Inside the head & \\
Missing & $29(20.6 \%)$ \\
Mery high frequency & $34(24.1 \%)$ \\
Nigh frequency & $60(42.6 \%)$ \\
Medium frequency & $11(7.8 \%)$ \\
Low frequency & $7(5.0 \%)$ \\
\hline
\end{tabular}

distress (BSI total score) and positively with emotional well-being (SF-36 emotional score). Only for the CTAQ-I subscale "activity engagement" significant positive correlations with physical well-being (SF-36) were found. The two subscales activity engagement and tinnitus willingness were weakly correlated to each other $(r=0.17$, $\mathrm{p}=0.045)$. Details are presented in Table IV.

\section{Correlation with clinical data}

Neither gender $(p=0.62)$ nor age $(p=0.68)$ correlated with the patients' acceptance levels. While no correla- tion between the CTAQ-I total score and the tinnitus duration $(\mathrm{p}=0.34)$ was found, acceptance was negatively correlated to the subjective tinnitus loudness $(r=-0.27$, $p=0.002)$ and the percentage of time patients were annoyed by their tinnitus $(\mathrm{r}=-0.57, \mathrm{p}<0.001)$. Additionally, the time patients were aware of their tinnitus was not correlated to their level of acceptance $(p=0.19)$.

Patients with a subjective hearing problem did not report significantly different CTQA-I scores than patients without subjective hearing problems $(\mathrm{p}=0.69)$. No differences were found across the different subjective pitch-level $(\mathrm{p}=0.82)$, and patients who experienced tinnitus with variable loudness did not significantly differ from those without varying tinnitus loudness $(\mathrm{p}=0.86)$. On the other hand, patients with hyperacusis reported lower acceptance scores than patients without hyperacusis $(66.4$ vs 80.2 points; $t=3.9, \mathrm{p}<0.001)$. Acceptance significantly predicted tinnitus distress $\left(\mathrm{R}^{2}=0.48\right.$, $\mathrm{b}=-0.64, \mathrm{p}<0.001)$, even after including hyperacusis in the model.

\section{Discussion}

The process of acceptance is increasingly gaining interest in the treatment of chronic tinnitus. Reliable and valid tools are needed to evaluate tinnitus acceptance in clinical practise and outcome research. The aim of the present study was to investigate the psychometric properties of the Italian version of the "Chronic Tinnitus Acceptance Questionnaire" (CTAQI) in a multicentre study in which 141 patients with tinnitus complaints from three study centres participated. The results of our study showed that the CTAQ-I is a reliable and valid measure for assessing the acceptance of chronic tinnitus.

To investigate the factorial structure of the CTAQ-I, we replicated the proposed two factor solution, but also calculated factor analyses with a three- and four-factor solution. The two-factor solution showed the clearest factor solution and accounted for $42.1 \%$ of the total variance; the results show an ever better fit than for the German version of the CTAQ. Although the 3- and 4-factor solutions explained slightly more variance, they also had several cross loadings and an inconclusive overall structure. We therefore decided to retain the original 2-factor solution. In conclusion, the results of our factor analyses support the two-factor structure, which is in line with the original pain acceptance questionnaire (CPAQ) ${ }^{32}$ and the two previous validations of the CTAQ in English and German, who also reported a two-factor solution ${ }^{29}$ (Moreland 2009, unpublished thesis).

The items that load on the factor "activity engagement", for example, include the full engagement in life (item 9: "I lead a full life even though I have tinnitus") and the pursuit of valu- 
Table II. Mean, standard deviations and variance of the CTAQ-I.

\begin{tabular}{|c|c|c|c|c|c|}
\hline & & Mean & SD & $\begin{array}{l}\text { Item-total } \\
\text { correlation }\end{array}$ & Missing \\
\hline 1. & I am getting on with the business of living no matter what my level of tinnitus is & 4.9 & 1.6 & 0.44 & - \\
\hline 2. & My life is going well, even though I have chronic tinnitus & 4.2 & 1.7 & 0.68 & $1.4 \%$ \\
\hline 3. & It's OK to experience ear noise & 2.3 & 2.2 & 0.24 & $2.8 \%$ \\
\hline 4. & I would gladly sacrifice important things in my life to control this tinnitus better* & 3.0 & 2.1 & 0.30 & $2.1 \%$ \\
\hline 5. & It's not necessary for me to control my ear noise in order to handle my life well & 3.0 & 2.2 & 0.45 & $0.7 \%$ \\
\hline 6. & Although things have changed, I am living a normal life despite my chronic tinnitus & 4.0 & 2.0 & 0.65 & $2.1 \%$ \\
\hline 7. & I need to concentrate on getting rid of my tinnitus* & 3.6 & 2.2 & 0.13 & - \\
\hline 8. & There are many activities I do when I experience tinnitus & 4.5 & 1.9 & 0.59 & - \\
\hline 9. & I lead a full life even though I have tinnitus & 4.3 & 1.8 & 0.57 & $0.7 \%$ \\
\hline 10. & Controlling tinnitus is less important than other goals in my life & 3.2 & 2.1 & 0.50 & - \\
\hline 11. & My thoughts and feelings about tinnitus must change before I can take important steps in my life* & 4.0 & 2.1 & 0.25 & $0.7 \%$ \\
\hline 12. & Despite the tinnitus, I am now sticking to a certain course in my life & 4.3 & 2.1 & 0.46 & $1.4 \%$ \\
\hline 13. & Keeping my tinnitus level under control takes first priority whenever I am doing something* & 3.6 & 2.1 & 0.49 & $0.7 \%$ \\
\hline 14. & Before I can make any serious plans, I have to get some control over my tinnitus* & 4.5 & 1.9 & 0.53 & - \\
\hline 15. & When my tinnitus increases, I can still take care of my responsibilities & 4.3 & 2.0 & 0.33 & - \\
\hline 16. & I will have better control over my life if I can control my negative thoughts about tinnitus* & 3.1 & 2.3 & 0.23 & - \\
\hline 17. & I avoid putting myself in situations where tinnitus might increase* & 3.0 & 2.4 & 0.44 & $0.7 \%$ \\
\hline 18. & My worries and fears about what tinnitus will do to me are true* & 3.2 & 2.2 & 0.57 & $0.7 \%$ \\
\hline 19. & It's a relief to realise that I don't have to change my tinnitus to get on with my life & 3.8 & 2.0 & 0.44 & $1.4 \%$ \\
\hline 19. & I have to struggle to do things when I have tinnitus* & 3.4 & 2.1 & 0.52 & - \\
\hline
\end{tabular}

"inversed item scores; Item-total correlations were corrected for the individual contribution of each item.

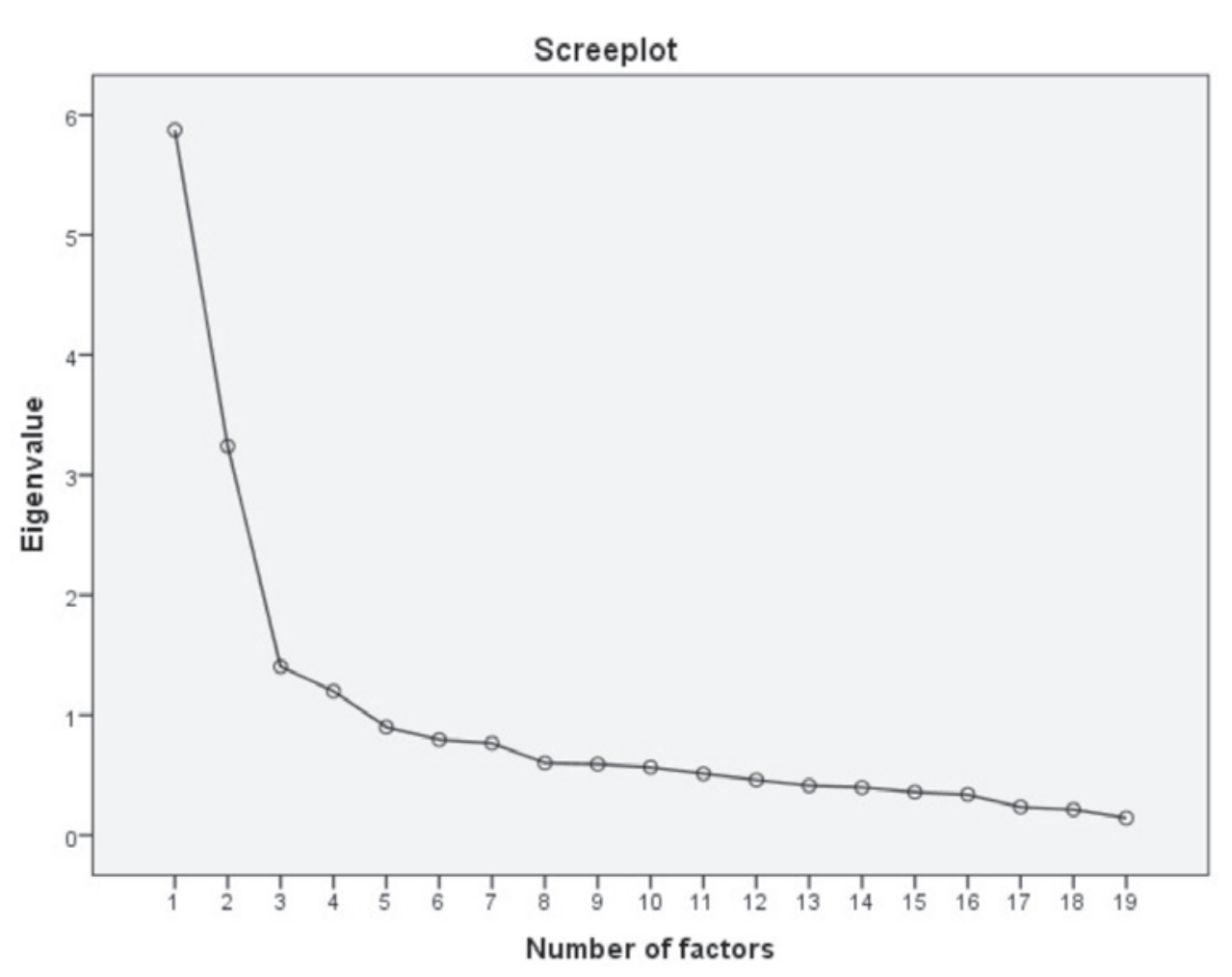

Fig. 1. Scree-Plot CTAQ-I. 
able everyday activities even if the tinnitus is present (item 8: "There are many activities I do when I experience tinnitus"). Other items assess the performing life's demands regardless of the perceived tinnitus level (item 1: "I am getting on with the business of living no matter what my level of tinnitus is"). The factor "tinnitus willingness" includes questions addressing the attempt to gain control over the perception of tinnitus itself (item 13: "Keeping my tinnitus level under control takes first priority whenever I am doing something"; item 14: "Before I can make any serious plan I have to get some control over my tinnitus"). Item 4 ("I would gladly sacrifice important things in my life to control this tinnitus better") depicted the extent to which some patients feel defeated by their tinnitus and how much they value even partial personal influence. Other questions that influence this factor capture attitudes and behaviours, such as the avoidance of situations where, from the patient's point of view, tinnitus may be worsening (item 17: "I avoid putting myself in situations where tinnitus might increase"), or the experienced need of controlling tinnitus related thoughts and emotions. The items of the factor "tinnitus willingness" clarify the central importance of overcoming tinnitus for many patients, and how much they align or change their behaviour accordingly.

Our results support McCracken et al.'s ${ }^{32}$ construction of ac- ceptance as involving both the giving up of the struggle to control tinnitus and pursuing normal life activities despite tinnitus. Nevertheless, the relationship between acceptance and control strategies requires further study ${ }^{20}$, as well as the potential benefits of control strategies and avoidance-based coping, by distinguishing between short- and long-term effects.

In our study the total score of the CTAQ-I as well as both subscales ("activity engagement" and "tinnitus willingness") showed good internal consistencies, which were comparable to the CTAQ-G ${ }^{29}$. Items 3 and 7 slightly lowered the internal consistency of the respective subscale. However, their exclusion would only result in mild improvement and the two items are profound parts of the theoretical concept of acceptance. Item 7 also showed the lowest item-total correlation of all items. In the previous validation of the German CTAQ-G item 7 showed an acceptable item-total correlation $(r=0.44)^{29}$, while no specific details were presented in the English validation (Moreland 2009, unpublished thesis). We therefore decided to keep both items in the questionnaire. Nevertheless, further research should critically review these items in larger international samples.

Construct validity was analysed by comparing the CTAQ-I with measures of tinnitus distress, psychological distress and

Table III. Results of the factor analysis (pattern matrix).

\begin{tabular}{|c|c|c|c|}
\hline & & & \\
\hline & & $\begin{array}{c}\text { Activity } \\
\text { engagement }\end{array}$ & $\begin{array}{c}\text { Tinnitus } \\
\text { willingness }\end{array}$ \\
\hline 1. & I am getting on with the business of living no matter what my level of tinnitus is & 0.68 & 0.02 \\
\hline 2. & My life is going well, even though I have chronic tinnitus & 0.76 & 0.32 \\
\hline 3. & It's OK to experience ear noise & 0.29 & 0.18 \\
\hline 4. & I would gladly sacrifice important things in my life to control this tinnitus better* & 0.01 & 0.56 \\
\hline 5. & It's not necessary for me to control my ear noise in order to handle my life well & 0.55 & 0.15 \\
\hline 6. & Although things have changed, I am living a normal life despite my chronic tinnitus & 0.79 & 0.26 \\
\hline 7. & I need to concentrate on getting rid of my tinnitus* & -0.35 & 0.25 \\
\hline 8. & There are many activities I do when I experience tinnitus & 0.81 & 0.18 \\
\hline 9. & I lead a full life even though I have tinnitus & 0.89 & 0.07 \\
\hline 10. & Controlling tinnitus is less important than other goals in my life & 0.59 & 0.21 \\
\hline 11. & My thoughts and feelings about tinnitus must change before I can take important steps in my life* & 0.03 & 0.44 \\
\hline 12. & Despite the tinnitus, I am now sticking to a certain course in my life & 0.63 & 0.11 \\
\hline 13. & Keeping my tinnitus level under control takes first priority whenever I am doing something* & 0.18 & 0.72 \\
\hline 14. & Before I can make any serious plans, I have to get some control over my tinnitus* & 0.25 & 0.73 \\
\hline 15. & When my tinnitus increases, I can still take care of my responsibilities & 0.51 & -0.01 \\
\hline 16. & I will have better control over my life if I can control my negative thoughts about tinnitus* & -0.08 & 0.51 \\
\hline 17. & I avoid putting myself in situations where tinnitus might increase* & 0.25 & 0.54 \\
\hline 18. & My worries and fears about what tinnitus will do to me are true* & 0.28 & 0.67 \\
\hline 19. & It's a relief to realise that I don't have to change my tinnitus to get on with my life & 0.57 & 0.09 \\
\hline 20. & I have to struggle to do things when I have tinnitus* & 0.27 & 0.61 \\
\hline
\end{tabular}

Extraction Method: maximum likelihood; Rotation Method: oblimin with Kaiser normalisation; bold values indicate which factor the item was assigned to; " inversed item scores. 
Table IV. Correlations between CTAQ-I, TQ 12-I, THI, BSI, and SF-36.

$\begin{array}{lccc} & \begin{array}{c}\text { CTAQ-I } \\ \text { total Score }\end{array} & \begin{array}{c}\text { CTAQ-I } \\ \text { activity engagement }\end{array} & -0.36^{*+*} \\ \text { tinnitus willingness }\end{array}$

quality of life. Increased tinnitus acceptance was associated with less tinnitus distress and less psychological distress, which is in line with previous studies on tinnitus acceptance 16202237 .

Furthermore, as indicated by previous research on tinnitus acceptance $22{ }^{37}$, higher levels of tinnitus acceptance were also related to an increased quality of life. While in our study emotional well-being was significantly correlated to the total score as well as both subscales, physical well-being seemed to be related only to activity engagement. This was not unexpected since the subscale 'activity engagement' includes a more physical aspect of acceptance, while tinnitus willingness may be understood as a more cognitive approach.

In our sample, the level of acceptance was neither correlated with gender or age, as also reported by Westin, Hayes ${ }^{19}$. Acceptance was negatively correlated with the subjective loudness of tinnitus, which is strongly related to the amount of tinnitus distress itself ${ }^{38}$. In a recent study, tinnitus acceptance fully mediated the relationship between the subjective tinnitus loudness and tinnitus distress, even when controlling for baseline depression and anxiety ${ }^{20}$. These results clearly indicate the role tinnitus acceptance may play in the understanding of subjective tinnitus distress.

We found no significant correlations between subjective hearing problems and tinnitus acceptance. As expected, patients with hyperacusis showed lower acceptance scores than patients without hyperacusis. This may be a fairly important finding, since hyperacusis is highly prevalent among patients with chronic tinnitus ${ }^{39}$, and is strongly related to tinnitus distress ${ }^{40}$, as well as to subjective tinnitus loudness and related comorbidities ${ }^{41}$. For clinical counselling, it might be highly relevant to assess the grade of acceptance or experiential avoidance, as symptom-related anxiety and protracted avoidance behaviour resulting from tinnitus and hyperacusis can both lead to disabling situations.
As shown in previous tinnitus studies ${ }^{42}$, the process of acceptance may also be influenced in treatments, which do not include specific acceptance-oriented methods (e.g. tinnitus counselling, Tinnitus Retraining Therapy (TRT) or elements of psychoeducation). Future tinnitus research should therefore focus on the conceptualisation of acceptance and experiential avoidance to increase the understanding of which factors may boost tinnitus acceptance within different types of treatments. The appraisal of tinnitus as a harmless - albeit disturbing - experience, may pave the way to acceptance of ear noise. The sensory experience of tinnitus cannot be separated from the emotional experience, and involves the senses, emotions, thoughts, actions and behaviours. Tinnitus patients with a pronounced state of suffering have not only to accept the tinnitus, but also come to terms with the tinnitusassociated cognitions and emotions, which play an important role in the acceptance of tinnitus. Patients may benefit from developing greater acceptance of thoughts and feelings associated with tinnitus ${ }^{18}$.

To capture such a wide concept as acceptance in future tinnitus research, it might be worth considering a combination of measures that reflect relevant features of acceptance, experiential avoidance and related variables. In addition to cognitive variables such as catastrophising, the inclusion of personality traits ${ }^{43}$ and tinnitus-related emotions may deepen our understanding of how patients cope with distressing tinnitus ${ }^{6}$. We assume that tinnitus-associated prolonged anger, shame as well as self-blame ${ }^{11}$, or the assignment of guilt to others, can also impair the development of an accepting attitude.

The CTAQ-I may be used as an assessment tool to evaluate the extent to which patients are able to adopt an accepting attitude towards tinnitus. Investigation of these aspects are worthwhile for better understanding helpful processes in tinnitus counselling, and is of practical use for further development of psychological therapies as well as for daily 
clinical work for all the various disciplines involved in tinnitus treatment.

\section{Limitations}

There are some potential sources of bias. The data was selfreported, collected as part of routine clinical work. Since our sample is a population from clinical practice, we can cautiously assume generalisability for outpatients. The sample consists exclusively of patients with chronic tinnitus; the significance of the results found is therefore limited for acute patients. The investigation has a cross-sectional design, so that any statements about cause-effect relationships or about factors influencing acceptance are not possible. We are not able to investigate the relationship between tinnitus acceptance and audiological data, since only self-report questionnaires were used. Future work is needed to clarify this aspect, as well as to investigate the test-retest reliability of the CTAQ-I.

\section{Conclusions}

In contemporary theories on how patients adapt to chronic diseases, acceptance is emerging as a valuable concept. Tinnitus acceptance is considered to be an important source of therapeutic change in behavioural-based treatments as well as in professional counselling for tinnitus. Further research is needed to highlight the importance of acceptance for adjusting to a life with chronic tinnitus and to deeper examine the clinical implications for the treatment of tinnitus. Our results show that the CTAQ-I is a reliable and valid instrument to measure the acceptance of patients suffering from chronic tinnitus. The CTAQ-I can also be adopted as an evaluation instrument for therapy research with tinnitus patients.

\section{Acknowledgements}

We thank COST Action BM1306 "TINNET - better understanding the heterogeneity of tinnitus to improve and develop new treatments" for supporting this study with a Short Term Scientific Mission to the Tinnitus Center in Rome, Italy. We thank Ryan Lundstrom for proofreading and language editing.

\section{Conflict of interest statement}

None declared.

\section{References}

1 Gopinath B, McMahon CM, Rochtchina E, et al. Incidence, persistence and progression of tinnitus symptoms in older adults: the Blue Mountains Hearing Study. Ear Hear 2010;31:407-12.
2 McCormack A, Edmondson-Jones M, Fortnum H, et al. The prevalence of tinnitus and the relationship with neuroticism in a middleaged UK population. J Psychosom Res 2014;76:56-60.

3 Milerová J, Anders M, Dvořák T, et al. The influence of psychological factors on tinnitus severity. Gen Hosp Psychiatry 2013;35:412-6.

4 Cronlein T, Langguth B, Pregler M, et al. Insomnia in patients with chronic tinnitus: cognitive and emotional distress as moderator variables. J Psychosom Res 2016;83:65-8.

5 Bartels H, Middel BL, van der Laan BF, et al. The additive effect of co-occurring anxiety and depression on health status, quality of life and coping strategies in help-seeking tinnitus sufferers. Ear Hear 2008;29:947-56.

6 Cima RF, Crombez G, Vlaeyen JW. Catastrophizing and fear of tinnitus predict quality of life in patients with chronic tinnitus. Ear Hear 2011;32:634-41.

7 Weidt S, Delsignore A, Meyer M, et al. Which tinnitus-related characteristics affect current health-related quality of life and depression? A cross-sectional cohort study. Psychiatry Res 2016;237:114-21.

8 Henry JA, Meikle MB. Psychoacoustic measures of tinnitus. J Am Acad Audiol 2000;11:138-55.

9 Ooms E, Vanheule S, Meganck R, et al. Tinnitus severity and its association with cognitive and somatic anxiety: a critical study. Eur Arch Otorhinolaryngol 2012;269:2327-33.

10 Durai M, Searchfield G. Anxiety and depression, personality traits relevant to tinnitus: a scoping review. Int J Audiol 2016;55:605-15.

11 Trevis KJ, McLachlan NM, Wilson SJ. Psychological mediators of chronic tinnitus: the critical role of depression. J Affect Disord 2016;204:234-40.

12 Ziai K, Moshtaghi O, Mahboubi H, et al. Tinnitus patients suffering from anxiety and depression: a review. Int Tinnitus J 2017;21:68-73.

13 Mazurek B, Szczepek AJ, Brüggemann P. Tinnitus - clinical symptoms and therapy. Laryngorhinootologie 2017;96:47-59.

14 Martinez-Devesa P, Perera R, Theodoulou M, et al. Cognitive behavioural therapy for tinnitus. Cochrane Database Syst Rev 2010:CD005233.

15 Hesser $\mathrm{H}$, Weise $\mathrm{C}$, Westin $\mathrm{V}$, et al. A systematic review and metaanalysis of randomized controlled trials of cognitive-behavioral therapy for tinnitus distress. Clin Psychol Rev 2011;31:545-53.

16 Westin V, Schulin M, Hesser H, et al. Acceptance and commitment therapy versus Tinnitus Retraining Therapy in the treatment of tinnitus: a randomised controlled trial. Behav Res Ther 2011;49:737-47.

17 Philippot P, Nef F, Clauw L, et al. A randomized controlled trial of mindfulness-based cognitive therapy for treating tinnitus. Clin Psychol Psychother 2012;19:411-9.

18 McKenna L, Marks EM, Vogt F. Mindfulness-based cognitive therapy for chronic Tinnitus: evaluation of benefits in a large sample of patients attending a Tinnitus clinic. Ear Hear 2018;39:359-66.

19 Westin V, Hayes SC, Andersson G. Is it the sound or your relationship to it? The role of acceptance in predicting tinnitus impact. Behav Res Ther 2008;46:1259-65.

20 Hesser H, Bånkestad E, Andersson G. Acceptance of Tinnitus as an independent correlate of Tinnitus severity. Ear Hear 2015;36:e176-e82.

21 Moring J, Bowen A, Thomas J, et al. Acceptance mediates the relationship between Tinnitus-related cognitions and anxiety sensitivity. Am J Audiol 2015;24:235-42.

22 Hesser H, Gustafsson T, Lundén C, et al. A randomized controlled trial of internet-delivered cognitive behavior therapy and acceptance and commitment therapy in the treatment of tinnitus. J Consult Clin Psychol 2012;80:649-61. 
23 Brandtstädter J, Renner G. Tenacious goal pursuit and flexible goal adjustment: explication and age-related analysis of assimilative and accommodative strategies of coping. Psychol Aging 1990;5:58-67.

24 Kleinstäuber M, Jasper K, Schweda I, et al. The role of fear-avoidance cognitions and behaviors in patients with chronic tinnitus. Cogn Behav Ther 2013;42:84-99.

25 McKenna L, Irwin R. Sound therapy for tinnitus - sacred cow or idol worship? An investigation of the evidence. Audiol Med 2008;6:16-24.

26 Dauman N, Erlandsson SI, Albarracin D, et al. Exploring tinnitusinduced disablement by persistent frustration in aging individuals: A grounded theory study. Front Aging Neurosci 2017;9:272.

27 Andersson G, Westin V. Understanding tinnitus distress: introducing the concepts of moderators and mediators. Int $\mathrm{J}$ Audiol 2008;47(Suppl 2):S106-S11.

28 Hesser H, Andersson G. The role of anxiety sensitivity and behavioral avoidance in tinnitus disability. Int J Audiol 2009;48:295-9.

29 Riedl D, Rumpold G, Schmidt A, et al. [Acceptance of tinnitus: Validation of the 'Akzeptanzfragebogen bei chronischem Tinnitus' (AFCT)] German. Laryngo- Rhino- Otologie 2014;93:840-7.

30 Langguth B, Goodey R, Azevedo A, et al. Consensus for tinnitus patient assessment and treatment outcome measurement: Tinnitus Research Initiative meeting, Regensburg, July 2006. Prog Brain Res 2007;166:525-36.

31 Bernini O, Pennato T, Cosci F, et al. The psychometric properties of the chronic pain acceptance questionnaire in Italian patients with chronic pain. J Health Psychol 2010;15:1236-45.

32 McCracken LM, Vowles KE, Eccleston C. Acceptance of chronic pain: component analysis and a revised assessment method. Pain 2004;107:159-66.

33 Passi S, Ralli G, Capparelli E, et al. The THI questionnaire: psychometric data for reliability and validity of the Italian version. Int Tinnitus J 2008;14:26-33.
34 Moschen R, Fioretti A, Eibenstein A, et al. Validation of the Italian Tinnitus Questionnaire Short Form (TQ 12-I) as a Brief Test for the Assessment of Tinnitus-Related Distress: Results of a Cross-Sectional Multicenter-Study. Front Psycho 2018;9:65

35 De Leo D, Frisoni GB, Rozzini R, et al. Italian community norms for the Brief Symptom Inventory in the elderly. Br J Clin Psychol 1993;32 ( Pt 2):209-13.

36 Apolone G, Mosconi P. The Italian SF-36 Health Survey: translation, validation and norming. J Clin Epidemiol 1998;51:102536.

37 Riedl D, Rumpold G, Schmidt A, et al. The influence of tinnitus acceptance on the quality of life and psychological distress in patients with chronic tinnitus. Noise Health 2015;17:374-81.

38 Hoekstra CEL, Wesdorp FM, van Zanten GA. Socio-demographic, health, and tinnitus related variables affecting tinnitus severity. Ear Hear 2014;35:544-54.

39 Landgrebe M, Zeman F, Koller M, et al. The Tinnitus Research Initiative (TRI) database: a new approach for delineation of tinnitus subtypes and generation of predictors for treatment outcome. BMC Med Inform Decis Mak 2010;10:42.

40 Schecklmann M, Lehner A, Schlee W, et al. Validation of screening questions for hyperacusis in chronic tinnitus. Biomed Res Int 2015;2015:191479.

${ }^{41}$ Kojima T, Kanzaki S, Oishi N, et al. Clinical characteristics of patients with tinnitus evaluated with the Tinnitus Sample Case History Questionnaire in Japan: a case series. Plos One 2017;12:e0180609-e.

42 Westin V, Östergren R, Andersson G. The effects of acceptance versus thought suppression for dealing with the intrusiveness of tinnitus. Int J Audiol 2008;47:112-8.

43 Kleinstauber M, Weise C, Andersson G, et al. Personality traits predict and moderate the outcome of Internet-based cognitive behavioural therapy for chronic tinnitus. Int J Audiol 2018;57:538-44.

Received: March 8, 2018 - Accepted: September 4, 2018

How to cite this article: Moschen R, Fioretti A, Eibenstein A, et al. Validation of the Chronic Tinnitus Acceptance Questionnaire (CTAQ-I): the Italian version. Acta Otorhinolaryngol Ital 2019;39:107-116. https://doi.org/10.14639/0392-100X-2144

Address for correspondence: David Riedl, University Clinic of Medical Psychology, Medical University of Innsbruck, Speckbacherstraße 23/3, 6020 Innsbruck, Austria. Tel. +43 512 504-26 231. Fax +43 512 504-26 232. E-mail: david.riedl@ tirol-kliniken.at 